\title{
Inclusion relationships for certain classes of analytic functions involving the Choi-Saigo-Srivastava operator
}

Nak Eun Cho ${ }^{1}$ and Min Yoon ${ }^{*}$

\section{"Correspondence:}

myoon@pknu.ac.kr

${ }^{2}$ Department of Statistics, Pukyong

National University, 45 Yongso-ro,

Busan, Korea

Full list of author information is

available at the end of the article

\begin{abstract}
The purpose of the present paper is to investigate some inclusion properties of certain classes of analytic functions associated with a family of linear operators which are defined by means of the Hadamard product (or convolution). Some invariant properties under convolution are also considered for the classes presented here. The results presented here include several previous known results as their special cases.

MSC: $30 C 45$

Keywords: subordination; Hadamard product (or convolution); starlike function; convex function; close-to-convex function; linear operator; Choi-Saigo-Srivastava operator; Carlson-Shaffer operator; Ruscheweyh derivative operator
\end{abstract}

\section{Introduction}

Let $\mathcal{A}$ denote the class of functions of the form

$$
f(z)=z+\sum_{k=2}^{\infty} a_{k} z^{k}
$$

which are analytic in the open unit disk $\mathbb{U}=\{z \in \mathbb{C}:|z|<1\}$. If $f$ and $g$ are analytic in $\mathbb{U}$, we say that $f$ is subordinate to $g$, written $f \prec g$ or $f(z) \prec g(z)$, if there exists an analytic function $w$ in $\mathbb{U}$ with $w(0)=0$ and $|\omega(z)|<1$ for $z \in \mathbb{U}$ such that $f(z)=g(\omega(z))$. We denote by $\mathcal{S}^{*}$, $\mathcal{K}$ and $\mathcal{C}$ the subclasses of $\mathcal{A}$ consisting of all analytic functions which are, respectively, starlike, convex and close-to-convex in $\mathbb{U}$ (see, e.g., Srivastava and Owa [1]).

Let $\mathcal{N}$ be a class of all functions $\phi$ which are analytic and univalent in $\mathbb{U}$ and for which $\phi(\mathbb{U})$ is convex with $\phi(0)=1$ and $\operatorname{Re}\{\phi(z)\}>0$ for $z \in \mathbb{U}$.

Making use of the principle of subordination between analytic functions, many authors investigated the subclasses $\mathcal{S}^{*}(\eta ; \phi), \mathcal{K}(\eta ; \phi)$ and $\mathcal{C}(\eta, \beta ; \phi, \psi)$ of the class $\mathcal{A}$ for $0 \leq \eta, \beta<1$, $\phi, \psi \in \mathcal{N}(c f .[2]$ and [3]), which are defined by

$$
\begin{aligned}
& \mathcal{S}^{\prime \prime}(\eta ; \phi):=\left\{f \in \mathcal{A}: \frac{1}{1-\eta}\left(\frac{z f^{\prime}(z)}{f(z)}-\eta\right) \prec \phi(z) \text { in } \mathbb{U}\right\}, \\
& \mathcal{K}(\eta ; \phi):=\left\{f \in \mathcal{A}: \frac{1}{1-\eta}\left(1+\frac{z f^{\prime \prime}(z)}{f^{\prime}(z)}-\eta\right) \prec \phi(z) \text { in } \mathbb{U}\right\}
\end{aligned}
$$

(0) 2013 Cho and Yoon; licensee Springer. This is an Open Access article distributed under the terms of the Creative Commons Attribution License (http://creativecommons.org/licenses/by/2.0), which permits unrestricted use, distribution, and reproduction in any medium, provided the original work is properly cited. 
and

$$
\mathcal{C}(\eta, \beta ; \phi, \psi):=\left\{f \in \mathcal{A}: \exists g \in \mathcal{S}^{*}(\eta ; \phi) \text { s.t. } \frac{1}{1-\beta}\left(\frac{z f^{\prime}(z)}{g(z)}-\beta\right) \prec \psi(z) \text { in } \mathbb{U}\right\} .
$$

For $\phi(z)=\psi(z)=(1+z) /(1-z)$ in the definitions above, we have the well-known classes $\mathcal{S}^{*}, \mathcal{K}$ and $\mathcal{C}$, respectively. Furthermore, for the function classes $\mathcal{S}^{*}[A, B]$ and $\mathcal{K}[A, B]$ investigated by Janowski ([4], also see [5]), it is easily seen that

$$
\mathcal{S}^{*}\left(0 ; \frac{1+A z}{1+B z}\right)=\mathcal{S}^{*}[A, B] \quad(-1 \leq B<A \leq 1)
$$

and

$$
\mathcal{K}\left(0 ; \frac{1+A z}{1+B z}\right)=\mathcal{K}[A, B] \quad(-1 \leq B<A \leq 1)
$$

We now define the function $\phi(a, c ; z)$ by

$$
\phi(a, c ; z):=\sum_{k=0}^{\infty} \frac{(a)_{k}}{(c)_{k}} z^{k+1} \quad\left(z \in \mathbb{U} ; a \in \mathbb{R} ; c \in \mathbb{R} \backslash \mathbb{Z}_{0}^{-} ; \mathbb{Z}_{0}^{-}:=\{-1,-2, \ldots\}\right),
$$

where $(v)_{k}$ is the Pochhammer symbol (or the shifted factorial) defined (in terms of the gamma function) by

$$
(v)_{k}:=\frac{\Gamma(v+k)}{\Gamma(v)}= \begin{cases}1 & \text { if } k=0 \text { and } v \in \mathbb{C} \backslash\{0\} \\ v(v+1) \cdots(v+k-1) & \text { if } k \in \mathbb{N}:=\{1,2, \ldots\} \text { and } v \in \mathbb{C}\end{cases}
$$

We also denote by $L(a, c): \mathcal{A} \rightarrow \mathcal{A}$ the operator defined by

$$
L(a, c) f(z)=\phi(a, c ; z) * f(z) \quad(z \in \mathbb{U})(z \in \mathbb{U} ; f \in \mathcal{A}),
$$

where the symbol $(*)$ stands for the Hadamard product (or convolution). Then it is easily observed from definitions (1.1) and (1.2) that

$$
L(2,1) f(z)=z f^{\prime}(z) \quad \text { and } \quad L(n+1,1) f(z)=D^{n} f(z) \quad(n>-1)
$$

where the symbol $D^{n}$ denotes the familiar Ruscheweyh derivative [6] (also, see [7]) for $n \in \mathbb{N}_{0}:=\mathbb{N} \cup\{0\}$. The operator $L(a, c)$, introduced and studied by Carlson-Shaffer [8], has been used widely on the space of analytic and univalent functions in $\mathbb{U}$ (see also [9]). Corresponding to the function $\phi(a, c ; z)$ defined by $(1.1)$, we also introduce a function $\phi_{\lambda}(a, c ; z)$ given by

$$
\phi(a, c ; z) * \phi_{\lambda}(a, c ; z)=\frac{z}{(1-z)^{\lambda}} \quad(\lambda>0)
$$

Analogous to $L(a, c)$, we now define the linear operator $\mathcal{I}_{\lambda}(a, c)$ on $\mathcal{A}$ as follows:

$$
\mathcal{I}_{\lambda}(a, c) f(z)=\phi_{\lambda}(a, c ; z) * f(z) \quad\left(a, c \in \mathbb{R} \backslash \mathbb{Z}_{0}^{-} ; \lambda>0 ; z \in \mathbb{U} ; f \in \mathcal{A}\right) .
$$


It is easily verified from definition (1.4) that

$$
\mathcal{I}_{2}(2,1) f(z)=f(z) \quad \text { and } \quad \mathcal{I}_{2}(1,1) f(z)=z f^{\prime}(z) .
$$

In particular, the operator $\mathcal{I}_{\lambda}(\mu+1,1)(\lambda>0 ; \mu>-1)$ was introduced by Choi et al. [2] who investigated (among other things) several inclusion properties involving various subclasses of analytic and univalent functions. For $\mu=n\left(n \in \mathbb{N}_{0}\right)$ and $\lambda=2$, we also note that the Choi-Saigo-Srivastava operator $\mathcal{I}_{\lambda}(\mu+1,1) f$ is the Noor integral operator of $n$th order of $f$ studied by Liu [10] and Noor et al. [11-15].

By using the operator $\mathcal{I}_{\lambda}(a, c)$, we introduce the following classes of analytic functions for $\phi, \psi \in \mathcal{N}, a, c \in \mathbb{R} \backslash \mathbb{Z}_{0}^{-}, \lambda>0$ and $0 \leq \eta, \beta<1$ :

$$
\begin{aligned}
& \mathcal{S}_{a, c}^{\lambda}(\eta ; \phi):=\left\{f \in \mathcal{A}: \mathcal{I}_{\lambda}(a, c) f(z) \in \mathcal{S}^{*}(\eta ; \phi)\right\}, \\
& \mathcal{K}_{a, c}^{\lambda}(\eta ; \phi):=\left\{f \in \mathcal{A}: \mathcal{I}_{\lambda}(a, c) f(z) \in \mathcal{K}^{*}(\eta ; \phi)\right\},
\end{aligned}
$$

and

$$
\mathcal{C}_{a, c}^{\lambda}(\eta, \beta ; \phi, \psi):=\left\{f \in \mathcal{A}: \mathcal{I}_{\lambda}(a, c) f(z) \in \mathcal{C}^{\prime \prime}(\eta, \beta ; \phi, \psi)\right\}
$$

We also note that

$$
f(z) \in \mathcal{K}_{a, c}^{\lambda}(\eta ; \phi) \quad \Longleftrightarrow \quad z f^{\prime}(z) \in \mathcal{S}_{a, c}^{\lambda}(\eta ; \phi) .
$$

In particular, we set

$$
\mathcal{S}_{a, c}^{\lambda}\left(\eta ; \frac{1+A z}{1+B z}\right)=\mathcal{S}_{a, c}^{\lambda}[\eta ; A, B] \quad(0 \leq \eta<1 ;-1 \leq B<A \leq 1)
$$

and

$$
\mathcal{K}_{a, c}^{\lambda}\left(\eta ; \frac{1+A z}{1+B z}\right)=\mathcal{K}_{a, c}^{\lambda}[\eta ; A, B] \quad(0 \leq \eta<1,-1 \leq B<A \leq 1) .
$$

Recently, Sokoł [16] extended the results given by Choi et al. [2] making use of some interesting proof techniques due to Ruscheweyh [17] and Ruscheweyh and Sheil-Small [18]. In this paper, we investigate several inclusion properties of the classes $\mathcal{S}_{a, c}^{\lambda}(\eta ; \phi), \mathcal{K}_{a, c}^{\lambda}(\eta ; \phi)$ and $\mathcal{C}_{a, c}^{\lambda}(\eta, \beta ; \phi, \psi)$. The integral-preserving properties in connection with the operator $\mathcal{I}_{\lambda}(a, c)$ are also considered. Furthermore, relevant connections of the results presented here with those obtained in earlier works are pointed out.

\section{Inclusion properties involving the operator $\mathcal{I}_{\lambda}(a, c)$}

The following lemmas will be required in our investigation.

Lemma 2.1 Let $\phi_{\lambda_{i}}(a, c ; z), \phi_{\lambda}\left(a_{i}, c ; z\right)$ and $\phi_{\lambda}\left(a, c_{i} ; z\right)(i=1,2)$ be defined by (1.3). Then, for $\lambda_{i}>0, a_{i}, c_{i} \in \mathbb{R} \backslash \mathbb{Z}_{0}^{-}(i=1,2)$,

$$
\begin{aligned}
& \phi_{\lambda_{1}}(a, c ; z)=\phi_{\lambda_{2}}(a, c ; z) * f_{\lambda_{1}, \lambda_{2}}(z), \\
& \phi_{\lambda}\left(a_{2}, c ; z\right)=\phi_{\lambda}\left(a_{1}, c ; z\right) * f_{a_{1}, a_{2}}(z),
\end{aligned}
$$


and

$$
\phi_{\lambda}\left(a, c_{1} ; z\right)=\phi_{\lambda}\left(a, c_{2} ; z\right) * f_{c_{1}, c_{2}}(z)
$$

where

$$
f_{s, t}(z)=\sum_{k=0}^{\infty} \frac{(s)_{k}}{(t)_{k}} z^{k+1} \quad(z \in \mathbb{U}) .
$$

Proof From definition (1.3), we know that

$$
\phi_{\lambda}(a, c ; z)=\sum_{k=0}^{\infty} \frac{(c)_{k}(\lambda)_{k}}{(a)_{k}(1)_{k}} z^{k+1} \quad(z \in \mathbb{U}) .
$$

Therefore, equations (2.1), (2.2) and (2.3) follow from (2.5) immediately.

Lemma 2.2 [17, pp.60-61] Let $t \geq s>0$. If $t \geq 2$ or $s+t \geq 3$, then the function defined by (2.4) belongs to the class $\mathcal{K}$.

Lemma 2.3 [18] Let $f \in \mathcal{K}$ and $g \in \mathcal{S}^{*}$. Then, for every analytic function $h$ in $\mathbb{U}$,

$$
\frac{(f * h g)(\mathbb{U})}{(f * g)(\mathbb{U})} \subset \overline{\operatorname{co}} h(\mathbb{U}),
$$

where $\overline{c o} h(\mathbb{U})$ denotes the closed convex hull of $h(\mathbb{U})$.

At first, the inclusion relationship involving the class $\mathcal{S}_{a, c}^{\lambda}(\eta ; \phi)$ is contained in Theorem 2.1 below.

Theorem 2.1 Let $\lambda_{2} \geq \lambda_{1}>0, a, c \in \mathbb{R} \backslash \mathbb{Z}_{0}^{-}, 0 \leq \eta<1$ and $\phi \in \mathcal{N}$. If $\lambda_{2} \geq 2$ or $\lambda_{1}+\lambda_{2} \geq 3$, then

$$
\mathcal{S}_{a, c}^{\lambda_{2}}(\eta ; \phi) \subset \mathcal{S}_{a, c}^{\lambda_{1}}(\eta ; \phi)
$$

Proof Let $f \in \mathcal{S}_{a, c}^{\lambda_{2}}(\eta ; \phi)$. From the definition of $\mathcal{S}_{a, c}^{\lambda_{2}}(\eta ; \phi)$, we have

$$
\frac{1}{1-\eta}\left(\frac{z\left(\mathcal{I}_{\lambda_{2}}(a, c) f(z)\right)^{\prime}}{\mathcal{I}_{\lambda_{2}}(a, c) f(z)}-\eta\right)=\phi(\omega(z)) \quad(z \in \mathbb{U})
$$

where $w$ is analytic in $\mathbb{U}$ with $|\omega(z)|<1(z \in \mathbb{U})$ and $w(0)=0$. By using (1.4), (2.1) and (2.6), we get

$$
\begin{aligned}
\frac{z\left(\mathcal{I}_{\lambda_{1}}(a, c) f(z)\right)^{\prime}}{\mathcal{I}_{\lambda_{1}}(a, c) f(z)} & =\frac{z\left(\phi_{\lambda_{1}}(a, c ; z) * f(z)\right)^{\prime}}{\phi_{\lambda_{1}}(a, c ; z) * f(z)}=\frac{z\left(\phi_{\lambda_{2}}(a, c ; z) * f_{\lambda_{1}, \lambda_{2}}(z) * f(z)\right)^{\prime}}{\phi_{\lambda_{2}}(a, c ; z) * f_{\lambda_{1}, \lambda_{2}}(z) * f(z)} \\
& =\frac{f_{\lambda_{1}, \lambda_{2}}(z) * z\left(\mathcal{I}_{\lambda_{2}}(a, c) f(z)\right)^{\prime}}{f_{\lambda_{1}, \lambda_{2}}(z) * \mathcal{I}_{\lambda_{2}}(a, c) f(z)} \\
& =\frac{f_{\lambda_{1}, \lambda_{2}}(z) *[(1-\eta) \phi(\omega(z))+\eta] \mathcal{I}_{\lambda_{2}}(a, c) f(z)}{f_{\lambda_{1}, \lambda_{2}}(z) * \mathcal{I}_{\lambda_{2}}(a, c) f(z)} .
\end{aligned}
$$


It follows from (2.6) and Lemma 2.2 that $\mathcal{I}_{\lambda_{2}}(a, c) f(z) \in \mathcal{S}^{*}$ and $f_{\lambda_{1}, \lambda_{2}} \in \mathcal{K}$, respectively. Let us put $s(\omega(z)):=(1-\eta) \phi(\omega(z))+\eta$. Then, by applying Lemma 2.3 to (2.7), we obtain

$$
\frac{\left\{f_{\lambda_{1}, \lambda_{2}} *[(1-\eta) \phi(w)+\eta] \mathcal{I}_{\lambda_{2}}(a, c) f\right\}}{\left\{f_{\lambda_{1}, \lambda_{2}} * \mathcal{I}_{\lambda_{2}}(a, c) f\right\}}(\mathbb{U}) \subset \overline{c o s}(w(\mathbb{U}))
$$

since $s$ is convex univalent. Therefore, from the definition of subordination and (2.8), we have

$$
\frac{1}{1-\eta}\left(\frac{z\left(\mathcal{I}_{\lambda_{1}}(a, c) f(z)\right)^{\prime}}{\mathcal{I}_{\lambda_{1}}(a, c) f(z)}-\eta\right) \prec \phi(z) \quad(z \in \mathbb{U}),
$$

or, equivalently, $f \in \mathcal{S}_{a, c}^{\lambda_{1}}(\eta ; \phi)$, which completes the proof of Theorem 2.1.

By using equations (1.4), (2.2) and (2.3), we have the following theorems.

Theorem 2.2 Let $\lambda>0, a_{2} \geq a_{1}>0, c \in \mathbb{R} \backslash \mathbb{Z}_{0}^{-}, 0 \leq \eta<1$ and $\phi \in \mathcal{N}$. If $a_{2} \geq 2$ or $a_{1}+a_{2} \geq 3$, then

$$
\mathcal{S}_{a_{1}, c}^{\lambda}(\eta ; \phi) \subset \mathcal{S}_{a_{2}, c}^{\lambda}(\eta ; \phi)
$$

Theorem 2.3 Let $\lambda>0, a \in \mathbb{R} \backslash \mathbb{Z}_{0}^{-}, c_{2} \geq c_{1}>0,0 \leq \eta<1$ and $\phi \in \mathcal{N}$. If $c_{2} \geq 2$ or $c_{1}+c_{2} \geq 3$, then

$$
\mathcal{S}_{a, c_{2}}^{\lambda}(\eta ; \phi) \subset \mathcal{S}_{a, c_{1}}^{\lambda}(\eta ; \phi) .
$$

Next, we prove the inclusion theorem involving the class $\mathcal{K}_{a, c}^{\lambda}(\eta ; \phi)$.

Theorem 2.4 Let $\lambda_{2} \geq \lambda_{1}>0, a, c \in \mathbb{R} \backslash \mathbb{Z}_{0}^{-}, 0 \leq \eta<1$ and $\phi \in \mathcal{N}$. If $\lambda_{2} \geq 2$ or $\lambda_{1}+\lambda_{2} \geq 3$, then

$$
\mathcal{K}_{a, c}^{\lambda_{2}}(\eta ; \phi) \subset \mathcal{K}_{a, c}^{\lambda_{1}}(\eta ; \phi) .
$$

Proof Applying (1.5) and Theorem 2.1, we observe that

$$
\begin{aligned}
f(z) \in \mathcal{K}_{a, c}^{\lambda_{2}}(\eta ; \phi) & \Longleftrightarrow \mathcal{I}_{\lambda_{2}}(a, c) f(z) \in \mathcal{K}(\eta ; \phi) \\
& \Longleftrightarrow z\left(\mathcal{I}_{\lambda_{2}}(a, c) f(z)\right)^{\prime} \in \mathcal{S}^{*}(\eta ; \phi) \\
& \Longleftrightarrow \mathcal{I}_{\lambda_{2}}(a, c)\left(z f^{\prime}(z)\right) \in \mathcal{S}^{*}(\eta ; \phi) \\
& \Longleftrightarrow z f^{\prime}(z) \in \mathcal{S}_{a, c}^{\lambda_{2}}(\eta ; \phi) \\
& \Longleftrightarrow z f^{\prime}(z) \in \mathcal{S}_{a, c}^{\lambda_{1}}(\eta ; \phi) \\
& \Longleftrightarrow \mathcal{I}_{\lambda_{1}}(a, c)\left(z f^{\prime}(z)\right) \in \mathcal{S}^{*}(\eta ; \phi) \\
& \Longleftrightarrow z\left(\mathcal{I}_{\lambda_{1}}(a, c) f(z)\right)^{\prime} \in \mathcal{S}^{*}(\eta ; \phi) \\
& \Longleftrightarrow \mathcal{I}_{\lambda_{1}}(a, c) f(z) \in \mathcal{K}(\eta ; \phi) \\
& \Longleftrightarrow f(z) \in \mathcal{K}_{a, c}^{\lambda_{1}}(\eta ; \phi),
\end{aligned}
$$

which evidently proves Theorem 2.4 . 
By using a similar method as in the proof of Theorem 2.4, we obtain the following two theorems.

Theorem 2.5 Let $\lambda>0, a_{2} \geq a_{1}>0, c \in \mathbb{R} \backslash \mathbb{Z}_{0}^{-}, 0 \leq \eta<1$ and $\phi \in \mathcal{N}$. If $a_{2} \geq 2$ or $a_{1}+a_{2} \geq 3$, then

$$
\mathcal{K}_{a_{1}, c}^{\lambda}(\eta ; \phi) \subset \mathcal{K}_{a_{2}, c}^{\lambda}(\eta ; \phi)
$$

Theorem 2.6 Let $\lambda>0, a \in \mathbb{R} \backslash \mathbb{Z}_{0}^{-}, c_{2} \geq c_{1}>0,0 \leq \eta<1$ and $\phi \in \mathcal{N}$. If $c_{2} \geq 2$ or $c_{1}+c_{2} \geq 3$, then

$$
\mathcal{K}_{a, c_{2}}^{\lambda}(\eta ; \phi) \subset \mathcal{K}_{a, c_{1}}^{\lambda}(\eta ; \phi)
$$

Taking $\phi(z)=(1+A z) /(1+B z)(-1 \leq B<A \leq 1 ; z \in \mathbb{U})$ in Theorems 2.1-2.6, we have the following corollaries below.

Corollary 2.1 Let $\lambda_{2} \geq \lambda_{1}>0$ and let $\lambda_{2} \geq \min \left\{2,3-\lambda_{1}\right\}$, and $a_{2} \geq a_{1}>0$ and $a_{2} \geq$ $\min \left\{2,3-a_{1}\right\}$. Then

$$
\mathcal{S}_{a_{1}, c}^{\lambda_{2}}[\eta ; A, B] \subset \mathcal{S}_{a_{1}, c}^{\lambda_{1}}[\eta ; A, B] \subset \mathcal{S}_{a_{2}, c}^{\lambda_{1}}[\eta ; A, B] \quad\left(c \in \mathbb{R} \backslash \mathbb{Z}_{0}^{-} ; 0 \leq \eta<1 ;-1 \leq B<A \leq 1\right),
$$

and

$$
\mathcal{K}_{a_{1}, c}^{\lambda_{2}}[\eta ; A, B] \subset \mathcal{K}_{a_{1}, c}^{\lambda_{1}}[\eta ; A, B] \subset \mathcal{K}_{a_{2}, c}^{\lambda_{1}}[\eta ; A, B] \quad\left(c \in \mathbb{R} \backslash \mathbb{Z}_{0}^{-} ; 0 \leq \eta<1 ;-1 \leq B<A \leq 1\right) .
$$

Corollary 2.2 Let $a_{2} \geq a_{1}>0$ and let $a_{2} \geq \min \left\{2,3-a_{1}\right\}$, and $c_{2} \geq c_{1}>0$ and $c_{2} \geq$ $\min \left\{2,3-c_{1}\right\}$. Then

$$
\mathcal{S}_{a_{1}, c_{2}}^{\lambda}[\eta ; A, B] \subset \mathcal{S}_{a_{1}, c_{1}}^{\lambda}[\eta ; A, B] \subset \mathcal{S}_{a_{2}, c_{1}}^{\lambda}[\eta ; A, B] \quad(\lambda>0 ; 0 \leq \eta<1 ;-1 \leq B<A \leq 1),
$$

and

$$
\mathcal{K}_{a_{1}, c_{2}}^{\lambda}[\eta ; A, B] \subset \mathcal{K}_{a_{1}, c_{1}}^{\lambda}[\eta ; A, B] \subset \mathcal{K}_{a_{2}, c_{1}}^{\lambda}[\eta ; A, B] \quad(\lambda>0 ; 0 \leq \eta<1 ;-1 \leq B<A \leq 1) .
$$

Corollary 2.3 Let $\lambda_{2} \geq \lambda_{1}>0$ and let $\lambda_{2} \geq \min \left\{2,3-\lambda_{1}\right\}$, and $c_{2} \geq c_{1}>0$ and $c_{2} \geq$ $\min \left\{2,3-c_{1}\right\}$. Then

$$
\mathcal{S}_{a, c_{2}}^{\lambda_{2}}[\eta ; A, B] \subset \mathcal{S}_{a, c_{1}}^{\lambda_{2}}[\eta ; A, B] \subset \mathcal{S}_{a, c_{1}}^{\lambda_{1}}[\eta ; A, B] \quad\left(a \in \mathbb{R} \backslash \mathbb{Z}_{0}^{-} ; 0 \leq \eta<1 ;-1 \leq B<A \leq 1\right)
$$

and

$$
\mathcal{K}_{a, c_{2}}^{\lambda_{2}}[\eta ; A, B] \subset \mathcal{K}_{a, c_{1}}^{\lambda_{2}}[\eta ; A, B] \subset \mathcal{K}_{a, c_{1}}^{\lambda_{1}}[\eta ; A, B] \quad\left(a \in \mathbb{R} \backslash \mathbb{Z}_{0}^{-} ; 0 \leq \eta<1 ;-1 \leq B<A \leq 1\right) .
$$

To prove the theorems below, we need the following lemma.

Lemma 2.4 [16] Let $\phi \in \mathcal{N}$. Iff $\in \mathcal{K}$ and $q \in \mathcal{S}^{*}(\eta ; \phi)$, then $f * q \in \mathcal{S}^{*}(\eta ; \phi)$. 
Proof Let $q \in \mathcal{S}^{*}(\eta ; \phi)$. Then

$$
z q^{\prime}(z)=[(1-\eta) \phi(\omega(z))+\eta] q(z) \quad(z \in \mathbb{U}),
$$

where $\omega$ is an analytic function in $\mathbb{U}$ with $|\omega(z)|<1(z \in \mathbb{U})$ and $w(0)=0$. Thus we have

$$
\frac{z(f(z) * q(z))^{\prime}}{f(z) * q(z)}=\frac{f(z) * z q^{\prime}(z)}{f(z) * q(z)}=\frac{f(z) *[(1-\eta) \phi(\omega(z))+\eta] q(z)}{f(z) * q(z)} \quad(z \in \mathbb{U}) .
$$

By using similar arguments to those used in the proof of Theorem 2.1, we conclude that (2.9) is subordinated to $\phi$ in $\mathbb{U}$ and so $f * q \in \mathcal{S}^{*}(\eta ; \phi)$.

Finally, we give the inclusion properties involving the class $\mathcal{C}_{a, c}^{\lambda}(\eta, \beta ; \phi, \psi)$.

Theorem 2.7 Let $\lambda_{2} \geq \lambda_{1}>0$ and $\lambda_{2} \geq \min \left\{2,3-\lambda_{1}\right\}$, and let $a_{2} \geq a_{1}>0$ and $a_{2} \geq$ $\min \left\{2,3-a_{1}\right\}$. Then

$$
\begin{aligned}
& \mathcal{C}_{a_{1}, c}^{\lambda_{2}}(\eta, \beta ; \phi, \psi) \subset \mathcal{C}_{a_{1}, c}^{\lambda_{1}}(\eta, \beta ; \phi, \psi) \subset \mathcal{C}_{a_{2}, c}^{\lambda_{1}}(\eta, \beta ; \phi, \psi) \\
& \left(c \in \mathbb{R} \backslash \mathbb{Z}_{0}^{-} ; 0 \leq \eta, \beta<1 ; \phi, \psi \in \mathcal{N}\right) .
\end{aligned}
$$

Proof We begin by proving that

$$
\mathcal{C}_{a_{1}, c}^{\lambda_{2}}(\eta, \beta ; \phi, \psi) \subset \mathcal{C}_{a_{1}, c}^{\lambda_{1}}(\eta, \beta ; \phi, \psi) .
$$

Let $f \in \mathcal{C}_{a_{1}, c}^{\lambda_{2}}(\eta, \beta ; \phi, \psi)$. Then there exists a function $q_{2} \in \mathcal{S}^{\prime \prime}(\eta ; \phi)$ such that

$$
\frac{1}{1-\beta}\left(\frac{z\left(\mathcal{I}_{\lambda_{2}}\left(a_{1}, c\right) f(z)\right)^{\prime}}{q_{2}(z)}-\beta\right) \prec \psi(z) \quad(z \in \mathbb{U}) .
$$

From (2.10), we obtain

$$
z\left(\mathcal{I}_{\lambda_{2}}\left(a_{1}, c\right) f(z)\right)^{\prime}=[(1-\beta) \psi(\omega(z))+\beta] q_{2}(z) \quad(z \in \mathbb{U}),
$$

where $w$ is an analytic function in $\mathbb{U}$ with $|\omega(z)|<1(z \in \mathbb{U})$ and $w(0)=0$. By virtue of (2.3), Lemma 2.2 and Lemma 2.4, we see that $f_{\lambda_{1}, \lambda_{2}}(z) * q_{2}(z) \equiv q_{1}(z)$ belongs to $\mathcal{S}^{*}(\eta ; \phi)$. Then, making use of (2.1), we have

$$
\begin{aligned}
\frac{1}{1-\beta}\left(\frac{z\left(\mathcal{I}_{\lambda_{1}}\left(a_{1}, c\right) f(z)\right)^{\prime}}{q_{1}(z)}-\beta\right) & =\frac{1}{1-\beta}\left(\frac{f_{\lambda_{1}, \lambda_{2}}(z) * z\left(\mathcal{I}_{\lambda_{2}}\left(a_{1}, c\right) f(z)\right)^{\prime}}{f_{\lambda_{1}, \lambda_{2}}(z) * q_{2}(z)}-\beta\right) \\
& =\frac{1}{1-\beta}\left(\frac{f_{\lambda_{1}, \lambda_{2}}(z) *[(1-\beta) \psi(\omega(z))+\beta] q_{2}(z)}{f_{\lambda_{1}, \lambda_{2}}(z) * q_{2}(z)}-\beta\right) \\
& \prec \psi(z) \quad(z \in \mathbb{U}) .
\end{aligned}
$$

Therefore we prove that $f \in \mathcal{C}_{a_{1}, c}^{\lambda_{1}}(\eta, \beta ; \phi, \psi)$.

For the second part, by using arguments similar to those detailed above with (2.2), we obtain

$$
\mathcal{C}_{a_{1}, c}^{\lambda_{1}}(\eta, \beta ; \phi, \psi) \subset \mathcal{C}_{a_{2}, c}^{\lambda_{1}}(\eta, \beta ; \phi, \psi)
$$

Thus the proof of Theorem 2.7 is completed. 
The following results can be obtained by using the same techniques as in the proof of Theorem 2.7, and so we omit the detailed proofs involved.

Theorem 2.8 Let $a_{2} \geq a_{1}>0$ and $a_{2} \geq \min \left\{2,3-a_{1}\right\}$, and let $c_{2} \geq c_{1}>0$ and $c_{2} \geq$ $\min \left\{2,3-c_{1}\right\}$. Then

$$
\begin{aligned}
& \mathcal{C}_{a_{1}, c_{2}}^{\lambda}(\eta, \beta ; \phi, \psi) \subset \mathcal{C}_{a_{1}, c_{1}}^{\lambda}(\eta, \beta ; \phi, \psi) \subset \mathcal{C}_{a_{2}, c_{1}}^{\lambda}(\eta, \beta ; \phi, \psi) \\
& (\lambda>0 ; 0 \leq \eta, \beta<1 ; \phi, \psi \in \mathcal{N}) .
\end{aligned}
$$

Theorem 2.9 Let $\lambda_{2} \geq \lambda_{1}>0$ and $\lambda_{2} \geq \min \left\{2,3-\lambda_{1}\right\}$, and let $c_{2} \geq c_{1}>0$ and $c_{2} \geq$ $\min \left\{2,3-c_{1}\right\}$. Then

$$
\begin{gathered}
\mathcal{C}_{a, c_{2}}^{\lambda_{2}}(\eta, \beta ; \phi, \psi) \subset \mathcal{C}_{a, c_{1}}^{\lambda_{2}}(\eta, \beta ; \phi, \psi) \subset \mathcal{C}_{a, c_{1}}^{\lambda_{1}}(\eta, \beta ; \phi, \psi) \\
\left(a \in \mathbb{R} \backslash \mathbb{Z}_{0}^{-} ; 0 \leq \eta, \beta<1 ; \phi, \psi \in \mathcal{N}\right) .
\end{gathered}
$$

Remark 2.1 (i) Taking $\lambda_{2}=\lambda_{1}+1\left(\lambda_{1} \geq 1\right), a_{2}=a_{1}+1\left(a_{1} \geq 1\right), c=1$ and $\eta=\beta=0$ in Theorems 2.1-2.2, Theorems 2.4-2.5 and Theorem 2.7, we have the results obtained by Choi et al. [2], which extend the results earlier given by Noor et al. [12, 14] and Liu [10].

(ii) For $a=\mu+1(\mu>-1), c=1$ and $\eta=\beta=0$, Theorems 2.1-2.2, Theorems 2.4-2.5 and Theorem 2.7 reduce the corresponding results obtained by Sokoł [16].

\section{Inclusion properties involving various operators}

The next theorem shows that the classes $\mathcal{S}_{a, c}^{\lambda}(\eta ; \phi), \mathcal{K}_{a, c}^{\lambda}(\eta ; \phi)$ and $\mathcal{C}_{a, c}^{\lambda}(\eta, \beta ; \phi, \psi)$ are invariant under convolution with convex functions.

Theorem 3.1 Let $\lambda>0, a>0, c \in \mathbb{R} \backslash \mathbb{Z}_{0}^{-}, 0 \leq \eta, \beta<1, \phi, \psi \in \mathcal{N}$ and let $g \in \mathcal{K}$. Then

(i) $f \in \mathcal{S}_{a, c}^{\lambda}(\eta ; \phi) \Longrightarrow g * f \in \mathcal{S}_{a, c}^{\lambda}(\eta ; \phi)$,

(ii) $f \in \mathcal{K}_{a, c}^{\lambda}(\eta ; \phi) \Longrightarrow g * f \in \mathcal{K}_{a, c}^{\lambda}(\eta ; \phi)$,

(iii) $f \in \mathcal{C}_{a, c}^{\lambda}(\eta, \beta ; \phi, \psi) \Longrightarrow g * f \in \mathcal{C}_{a, c}^{\lambda}(\eta, \beta ; \phi, \psi)$.

Proof (i) Let $f \in \mathcal{S}_{a, c}^{\lambda}(\eta ; \phi)$. Then we have

$$
\frac{1}{1-\eta}\left(\frac{z\left(\mathcal{I}_{\lambda}(a, c)(g * f)(z)\right)^{\prime}}{\mathcal{I}_{\lambda}(a, c)(g * f)(z)}-\eta\right)=\frac{1}{1-\eta}\left(\frac{g(z) * z\left(\mathcal{I}_{\lambda}(a, c) f(z)\right)^{\prime}}{g(z) * \mathcal{I}_{\lambda}(a, c) f(z)}-\eta\right) .
$$

By using the same techniques as in the proof of Theorem 2.1, we obtain (i).

(ii) Let $f \in \mathcal{K}_{a, c}^{\lambda}(\eta ; \phi)$. Then, by (1.5), $z f^{\prime}(z) \in \mathcal{S}_{a, c}^{\lambda}(\eta ; \phi)$ and hence from (i), $g(z) * z f^{\prime}(z) \in$ $\mathcal{S}_{a, c}^{\lambda}(\eta ; \phi)$. Since

$$
g(z) * z f^{\prime}(z)=z(g * f)^{\prime}(z),
$$

we have (ii) applying (1.5) once again.

(iii) Let $f \in \mathcal{C}_{a, c}^{\lambda}(\eta, \beta ; \phi, \psi)$. Then there exists a function $q \in \mathcal{S}^{*}(\eta ; \phi)$ such that

$$
z\left(\mathcal{I}_{\lambda}(a, c) f(z)\right)^{\prime}=[(1-\beta) \psi(\omega(z))+\beta] q(z) \quad(z \in \mathbb{U})
$$


where $w$ is an analytic function in $\mathbb{U}$ with $|\omega(z)|<1(z \in \mathbb{U})$ and $w(0)=0$. From Lemma 2.4, we have that $g * q \in \mathcal{S}^{*}(\eta ; \phi)$. Since

$$
\begin{aligned}
\frac{1}{1-\beta}\left(\frac{z\left(\mathcal{I}_{\lambda}(a, c)(g * f)(z)\right)^{\prime}}{(g * q)(z)}-\beta\right) & =\frac{1}{1-\beta}\left(\frac{g(z) * z\left(\mathcal{I}_{\lambda}(a, c) f(z)\right)^{\prime}}{g(z) * q(z)}-\beta\right) \\
& =\frac{1}{1-\beta}\left(\frac{g(z) *[(1-\beta) \psi(\omega(z))+\beta] q(z)}{g(z) * q(z)}-\beta\right) \\
& \prec \psi(z) \quad(z \in \mathbb{U}),
\end{aligned}
$$

we obtain (iii).

Now we consider the following operators defined by

$$
\Psi_{1}(z)=\sum_{k=1}^{\infty} \frac{1+c}{k+c} z^{k} \quad(\operatorname{Re}\{c\} \geq 0 ; z \in \mathbb{U})
$$

and

$$
\Psi_{2}(z)=\frac{1}{1-x} \log \left[\frac{1-x z}{1-z}\right] \quad(\log 1=0 ;|x| \leq 1, x \neq 1 ; z \in \mathbb{U}) .
$$

It is well known ([19], see also $[6,20])$ that the operators $\Psi_{1}$ and $\Psi_{2}$ are convex univalent in $\mathbb{U}$. Therefore we have the following result, which can be obtained from Theorem 3.1 immediately.

Corollary 3.1 Let $a>0, \lambda>0, c \in \mathbb{R} \backslash \mathbb{Z}_{0}^{-}, 0 \leq \eta, \beta<1, \phi, \psi \in \mathcal{N}$ and let $\Psi_{i}(i=1,2)$ be defined by (3.1) and (3.2). Then

(i) $f \in \mathcal{S}_{a, c}^{\lambda}(\eta ; \phi) \Longrightarrow \Psi_{i} * f \in \mathcal{S}_{a, c}^{\lambda}(\eta ; \phi)$,

(ii) $f \in \mathcal{K}_{a, c}^{\lambda}(\eta ; \phi) \Longrightarrow \Psi_{i} * f \in \mathcal{K}_{a, c}^{\lambda}(\eta ; \phi)$,

(iii) $f \in \mathcal{C}_{a, c}^{\lambda}(\eta, \beta ; \phi, \psi) \Longrightarrow \Psi_{i} * f \in \mathcal{C}_{a, c}^{\lambda}(\eta, \beta ; \phi, \psi)$.

Remark 3.1 Letting $a=\mu+1(\mu>-1), c=1$ and $\eta=\beta=0$ in Theorem 3.1, we have the corresponding results given by Sokol [16].

\section{Competing interests}

The authors declare that they have no competing interests.

\section{Authors' contributions}

All authors jointly worked on the results and they read and approved the final manuscript.

\section{Author details}

${ }^{1}$ Department of Applied Mathematics, Pukyong National University, 45 Yongso-ro, Busan, Korea. ${ }^{2}$ Department of Statistics, Pukyong National University, 45 Yongso-ro, Busan, Korea.

\section{Acknowledgements}

Dedicated to Professor Hari M Srivastava.

The authors would like to express their thanks to the referees for some valuable comments regarding a previous version of this paper. This research was supported by the Basic Science Research Program through the National Research

Foundation of Korea (NRF) funded by the Ministry of Education, Science and Technology (No. 2012-0002619). 


\section{References}

1. Srivastava, HM, Owa, S (eds.): Current Topics in Analytic Function Theory. World Scientific, Singapore (1992)

2. Choi, JH, Saigo, M, Srivastava, HM: Some inclusion properties of a certain family of integral operators. J. Math. Anal. Appl. 276, 432-445 (2002)

3. Ma, WC, Minda, D: An internal geometric characterization of strongly starlike functions. Ann. Univ. Mariae Curie-SkTodowska, Sect. A 45, 89-97 (1991)

4. Janowski, W: Some extremal problems for certain families of analytic functions. Bull. Acad. Pol. Sci., Sér. Sci. Math. Astron. Phys. 21, 17-25 (1973)

5. Goel, RM, Mehrok, BS: On the coefficients of a subclass of starlike functions. Indian J. Pure Appl. Math. 12, 634-647 (1981)

6. Ruscheweyh, S: New criteria for univalent functions. Proc. Am. Math. Soc. 49, 109-115 (1975)

7. Al-Amiri, HS: On Ruscheweyh derivatives. Ann. Pol. Math. 38, 88-94 (1980)

8. Carlson, BC, Shaffer, DB: Starlike and prestarlike hypergeometric functions. SIAM J. Math. Anal. 159, 737-745 (1984)

9. Srivastava, HM, Owa, S: Some characterizations and distortions theorems involving fractional calculus, generalized hypergeometric functions, Hadamard products, linear operators, and certain subclasses of analytic functions. Nagoya Math. J. 106, 1-28 (1987)

10. Liu, JL: The Noor integral and strongly starlike functions. J. Math. Anal. Appl. 261, 441-447 (2001)

11. Liu, JL, Noor, Kl: Some properties of Noor integral operator. J. Nat. Geom. 21, 81-90 (2002)

12. Noor, Kl: On new classes of integral operators. J. Nat. Geom. 16, 71-80 (1999)

13. Noor, Kl: Some classes of $p$-valent analytic functions defined by certain integral operator. Appl. Math. Comput. 157, 835-840 (2004)

14. Noor, Kl, Noor, MA: On integral operators. J. Math. Anal. Appl. 238, 341-352 (1999)

15. Noor, Kl, Noor, MA: On certain classes of analytic functions defined by Noor integral operator. J. Math. Anal. Appl. 281, 244-252 (2003)

16. Sokoł, J: Classes of analytic functions associated with the Choi-Saigo-Srivastava operator. J. Math. Anal. Appl. 318 , 517-525 (2006)

17. Ruscheweyh, S: Convolutions in Geometric Function Theory. Sem. Math. Sup., vol. 83. Presses University Montreal, Montreal (1982)

18. Ruscheweyh, S, Sheil-Small, T: Hadamard product of Schlicht functions and the Pólya-Schoenberg conjecture Comment. Math. Helv. 48, 119-135 (1975)

19. Barnard, RW, Kellogg, C: Applications of convolution operators to problems in univalent function theory. Mich. Math. J. 27, 81-93 (1980)

20. Owa, S, Srivastava, HM: Some applications of the generalized Libera integral operator. Proc. Jpn. Acad., Ser. A, Math Sci. 62, 125-128 (1986)

\section{Submit your manuscript to a SpringerOpen ${ }^{\ominus}$ journal and benefit from:}

- Convenient online submission

- Rigorous peer review

Immediate publication on acceptance

Open access: articles freely available online

- High visibility within the field

- Retaining the copyright to your article 